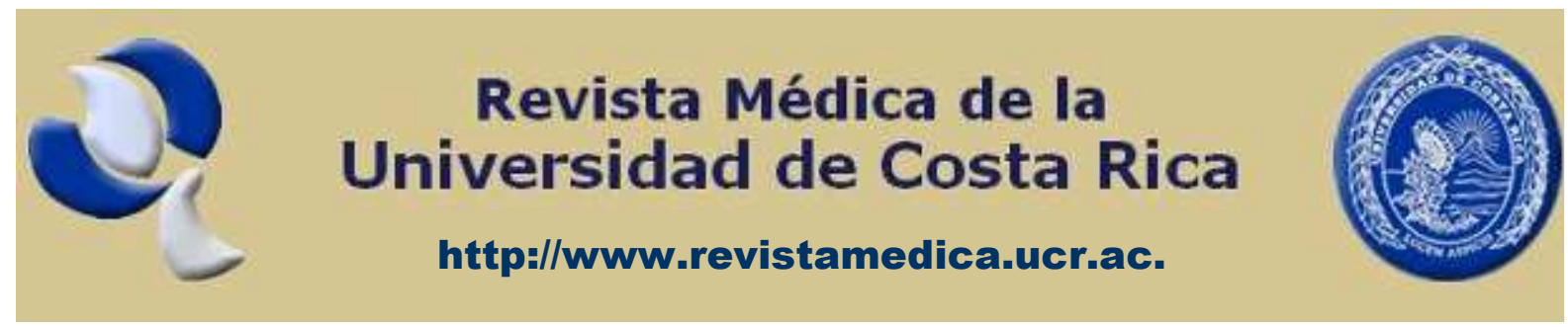

Investigación original

\title{
ESTUDIO ANATOMOPATOLÓGICO EN PLACENTAS DE MUJERES CON HIPERTENSIÓN INDUCIDA POR EL EMBARAZO
}

\author{
Zamora Vargas, Luis ${ }^{1}$; Aguilar Salazar, Cintya ${ }^{2}$ y Cascante Campos, Yancy ${ }^{3}$ \\ ${ }^{1}$ Médico-cirujano, esp. anatomía patológica, Dpto. anatomía, Escuela de Medicina, Universidad de Costa Rica \\ 2 Bach. En Biología, Universidad de Costa Rica, San Pedro, Costa Rica. \\ ${ }^{3}$ Médico, residente gineco-obstetricia, Hospital de la Mujer, San José, Costa Rica.
}

\begin{abstract}
Resumen
La hipertensión inducida por el embarazo (HIE) puede desembocar en una serie de síndromes dependiendo de la gravedad con que se presente y los signos y síntomas que lo acompañen. Sabemos que la interrupción del embarazo junto con la expulsión de la placenta termina con estos problemas. En total se estudiaron 28 placentas en donde los hallazgos más importantes fueron que el $100 \%$ de las muestras relacionadas a HIE presentaron patologías y el $87 \%$ de las muestras presentaron menos de un $50 \%$ de área placentaria funcional.
\end{abstract}

Palabras Clave: Hipertensión, pre-eclampsia, eclampsia, placenta, embarazo. Recibido: Marzo 2008. Aceptado: Marzo 2008. Publicado: Marzo 2008.

Revista electrónica publicada por el Departamento de Farmacología de la Escuela de Medicina de la Universidad de Costa Rica, 2060 San José, Costa Rica. ${ }^{\circledR}$ All rights

reserved. 


\begin{abstract}
The hypertension induced by pregnancy (HIP) can lead into a series of syndromes depending on the gravity with that you show up and signs and symptoms that accompany it. We know that the interruption of the pregnancy along with the expulsion of the placenta finishes with these problems. In total we studied 28 placentas where the more important findings were that $100 \%$ of the signs related to HIP presented pathologies and $87 \%$ of the signs they showed less than $50 \%$ of placental functional area.
\end{abstract}

Keywords: hypertension, pre-eclampsia, eclampsia, placenta, pregnancy.

\section{Introduction}

En la literatura mundial del 5 al $20 \%$ de los embarazos normales reportan desarrollo de hipertensión inducida por el embarazo (HIE) [1,15] que en ocasiones dependiendo de su severidad y otros síntomas y signos obliga a la interrupción del embarazo como medida terapéutica. En nuestro país, aunque no existen estudios en cuanto a su frecuencia, este fenómeno es observado. La HIE puede presentarse como pre-eclampsia, eclampsia y aparte el síndrome HELLP (del inglés: hemolysis, elevated liver enzymes y low platelet count). La pre-eclampsia es la más común de ellas [1,2], y desde 1916 cuando se definió esta enfermedad [2], no se ha logrado dilucidar las causas de su aparición. En la literatura mundial encontramos intentos en procura de dilucidar la fisiopatología de este

\section{Objetivo}

Siguiendo esta línea de pensamiento nuestro objetivo en el presente trabajo es el de revisar 28 placentas de madres cuyo diagnóstico de ingreso al Hospital padecimiento, concentrándose la mayoría en problemas del endotelio vascular [3,5], antioxidantes [6] 0 intermediarios en las respuesta inflamatoria [4]; sin embargo también se ha citado la isquemia placentaria como factor clave $[7,9,10,11,12,13,14]$. Creemos que el análisis de las anormalidades en la relación fetoplacentaria que ocasionen insuficiencias de los requerimientos del producto, puede ser el punto de partida de la investigación patofisiológicas de la HIE, en la medida en que se analice la capacidad placentaria de influir en la homeostasis materna, causando la hipertensión y sus complicaciones asociadas como proteinuria, reacción inflamatoria severa y daño hepático.

de la Mujer fue de HIE y HELLP, con la intención de reconocer cambios anatomopatológicos que impidan una adecuada función placentaria. 


\section{Método y Material}

Se estudiaron 28 placentas de mujeres cuyo único criterio de recolección fue el diagnóstico de ingreso confirmado de HIE y HELLP, que ingresaron en el Hospital de la Mujer. Se consignaron las edades gestacionales en el momento de la interrupción natural o provocado del embarazo, así como los pesos de los productos al nacer, los cuales se compararon con los pesos normales establecidos por Little [16]. Las placentas se conservaron en formalina Bufferizada al $10 \%$. Se les realizó un estudio macroscópico e histológico. Este último con Hematoxilina Eosina en bloques de parafina, para lo cual se tomaron muestras por cada tipo de hallazgo patológico macroscópico encontrado, con el fin de llegar a un diagnóstico anatomopatológico. En general se tomaron de 1 a 3 muestras por cada tipo de lesión, que representaron igual

\section{Resultados:}

Con respecto al peso de las placentas (tabla 1) una vez fijadas, se obtuvo que el $75 \%(21 / 28)$ de las placentas presentaban un peso menor a lo esperado en relación a las semanas de gestación; encontrándose una diferencia promedio de 133 gramos entre el peso esperado y el obtenido.

Como se observa en la tabla 1 el peso de los productos respecto al esperado es menor en 24/28 nacidos, correspondiendo a un $86 \%$ de los casos, obteniéndose una diferencia de peso promedio de 709 gramos entre el peso real y esperado. El porcentaje restante $(14 \%)$ de las placentas, obtuvieron número de bloques en parafina y láminas en hematoxilina eosina.

En el análisis macroscópico se tomaron en cuenta los siguientes aspectos: Peso de la placenta (fijada en formalina al $10 \%$ ) en balanza granataria de dos decimales, el cual se correlacionó con el peso normal esperado para la edad gestacional según Collen [8]. Así como de la implantación del cordón umbilical y registro de cualquier otro hallazgo patológico que indique algún grado de insuficiencia placentaria con su correspondiente estudio histopatológico.

Con la descripción detallada de los hallazgos anteriores se calculó cualitativamente un valor en porcentaje que se denominó área funcional placentaria (AFP). Este cálculo cualitativo se efectuó al restar el porcentaje aproximado de la suma de los daños placentarios descritos al volumen placentario total.

pesos mayores o cercanos al peso placentario esperado. Sin embargo, mostraron en promedio un $25 \%$ de AFP y llama la atención que esta disminución fue a expensas de membranas hialinas y fibrosis marginal más que a infartos.

En 9/28 se observó implantación velamentosa (Fig. 1E), lo que corresponde a un $23 \%$ de los casos. La fibrosis marginal (Fig. 1B), se encontró en $13 / 28$ casos representando un $46 \%$. Histológicamente la fibrosis marginal correspondió a depósito de material denso, fibroso (Fig. 1G), el $78 \%$ de estos casos tenían más de la mitad del perímetro placentario afectado. 
Se identificaron macroscópicamente depósitos blanquecinos en la cara materna de los cotiledones (Fig. 1A). Histológicamente estos depósitos correspondieron a material fibrinoide a manera de gruesas membranas hialinas en las vellosidades coriales (Fig. 1I). Este fenómeno se presento en 18/28 (64\%), de los cuales el $80 \%$ esta membrana cubría más de la mitad de la superficie de la placenta.

Los infartos se observaron en 25/28 casos lo que correspondió a un $89 \%$ de los casos, siendo recientes 23/28 (Fig. 1D), y antiguos 25/28 (Fig. 1C, $1 F$ y $1 \mathrm{H}$ ). Los infartos recientes y antiguos se caracterizaron por ser múltiples y en 9/28 incontables. En el $48 \%$ de estos

\section{Discusión}

En Costa Rica no se cuenta con un estudio anatomopatológico y/o clínico de pacientes con esta enfermedad. La literatura dedicada al estudio morfológico de las placentas de mujeres que desarrollaron HIE, no se refiere con detalle a los fenómenos encontrados en las placentas de nuestro estudio. Tampoco encontramos referencias a pesos normales de placentas o fetos para Costa Rica razón por la cual nos vimos obligados a utilizar datos internacionales. En el caso de los pesos de las placentas según Collen [8] ,del cual escogimos el percentil 50 ya que de acuerdo a nuestra experiencia de 20 años como patólogo en un hospital general, esta columna coincide más con los pesos observados en la práctica. En el caso del peso de los neonatos escogimos el trabajo de Little [16]. Los hallazgos que más incidieron en la disminución de las AFP fueron los siguientes: En primer lugar los infartos, casos se encontraron más de 12 infartos por placenta, abarcando más de la mitad de la superficie placentaria.

En cuanto al área funcional placentaria (AFP), 12/28 (43\%) presentaron un AFP de menos de 20\%; 8/28 (29\%) presentaron un estimación AFP entre $21-40 \%$; $4 / 28$ (14\%) entre $41-60 \%$ y de igual manera $4 / 28$ casos (14\%) tuvieron entre $61-80 \%$ de AFP estimada; 0/28 casos obtuvieron más de un $80 \%$ de AFP. Todas las pacientes con insuficiencia placentaria de más de un $50 \%$ presentaron preeclampsia severa y se observó que entre menos AFP mayor la severidad del cuadro de HIE (Tabla 2).

reconocidos macroscópica e histológicamente de 2 tipos, los antiguos como zonas de consistencia aumentada, cuya superficie de corte es dura y blanquecina, mostrándose histológicamente como áreas extensas de cicatrización, sustituyendo las vellosidades coriales; y los infartos recientes 0 hemorrágicos reconocidas como áreas de coloración rojo oscuro, reblandecidas, algunos inclusive con cavitación. Ambos tipos de infartos jugaron un papel importante en la disminución de la cantidad de vellosidades útiles. En segundo lugar la membrana hialina superficial, consignado de esta manera debido a su semejanza con lo observado en los pulmones de pacientes con enfermedad de membrana hialina; y por último la fibrosis marginal definida como una zona blanco grisáceo a manera de aro marginal en la periferia de la placenta. Tanto la fibrosis marginal como las

Revista electrónica publicada por el Departamento de Farmacología de la Escuela de Medicina de la Universidad de Costa Rica, 2060 San José, Costa Rica. ${ }^{\circledR}$ All rights reserved. 
membranas hialinas superficiales, funcionan como verdaderas barreras al ensanchar el espacio entre los lagos maternos y los vasos fetales de las vellosidades, con lo que el intercambio gaseoso y de otros productos necesarios para el feto no es eficiente.

En cuanto a los demás datos, tales como el peso de las placentas, también mostraron estar a favor de una insuficiencia placentaria de larga evolución, que junto con a lo anterior, incidieron en el bajo peso de los productos al nacer, aún en placentas que mostraron pesos semejantes 0 mayores a los esperados. En estas se mostró una severa disminución de su AFP a expensas de fibrosis marginal y presencia de membranas hialinas. Esto nos hace pensar que el bajo peso de las placentas se deba en parte a la cantidad de infartos observados.

Demostramos además, según lo expresado en la tabla 2, que la mayoría de las eclampsias severas, incluido el único caso de HELLP, se encuentran entre las placentas que mostraron una AFP del $20 \%$ o menos. Llama la atención que ninguna placentas obtuvo un AFP de $80 \%$ o más, lo que señala favorablemente nuestra hipótesis de que la insuficiencia placentaria juega un importante papel en la génesis de la HIE.

Eventualmente se podría pensar que los hallazgos descritos correspondan a consecuencias del HIE. Nuestro argumento más fuerte en demostrar que pueden ser parte de la causa y no consecuencias, es la cronología misma de las lesiones, ya que estas son de muy larga evolución (sobre todo los infartos antiguos) por lo que cronológicamente se instalaron antes del inicio de la HIE.

En este punto es necesario recordar que la placenta es al feto en desarrollo, lo que los pulmones, hígado y riñones son al neonato, por lo tanto si la placenta entra en insuficiencia, el daño al feto será de grandes dimensiones, pudiendo incluso llegar al fallecimiento. Creemos que los aspectos comentados anteriormente, deben ser analizados cuidadosamente en cuanto a la fisiopatología de la HIE, en el sentido de que una placenta insuficiente (infartada y fibrótica), podría ser capaz de enviar algún tipo de señal dirigida a un aumento de la presión arterial y de la permeabilidad vascular materna, con el fin de neutralizar la insuficiencia placentaria, en detrimento de la homeostasis materna y a favor del producto (recordemos la capacidad endocrina placentaria reconocida últimamente). Un dato más que sugiere que la placenta esta involucrada de alguna forma con el desarrollo de la HIE, es que una vez retirada la placenta sin importar la edad gestacional, las madres regresan a su estado de normalidad. 

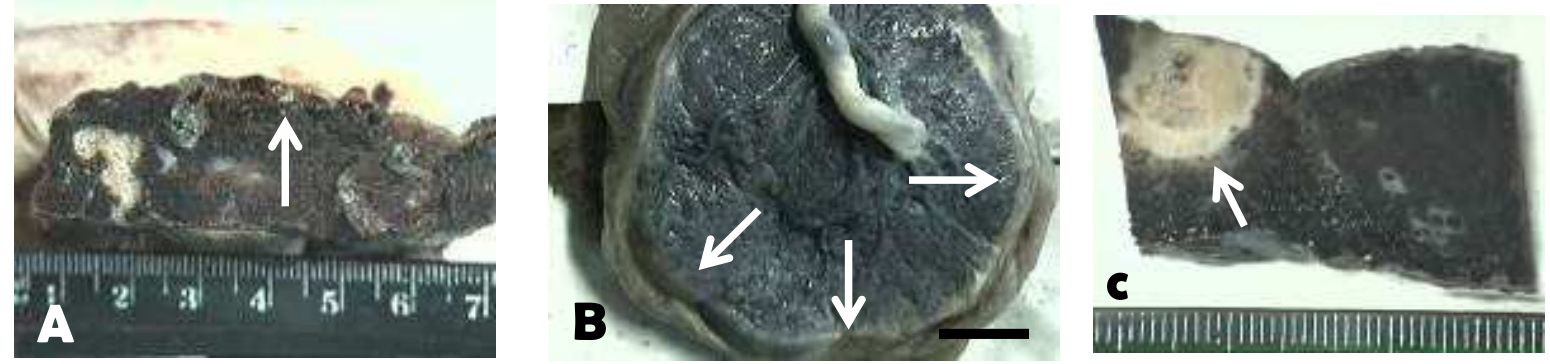

C
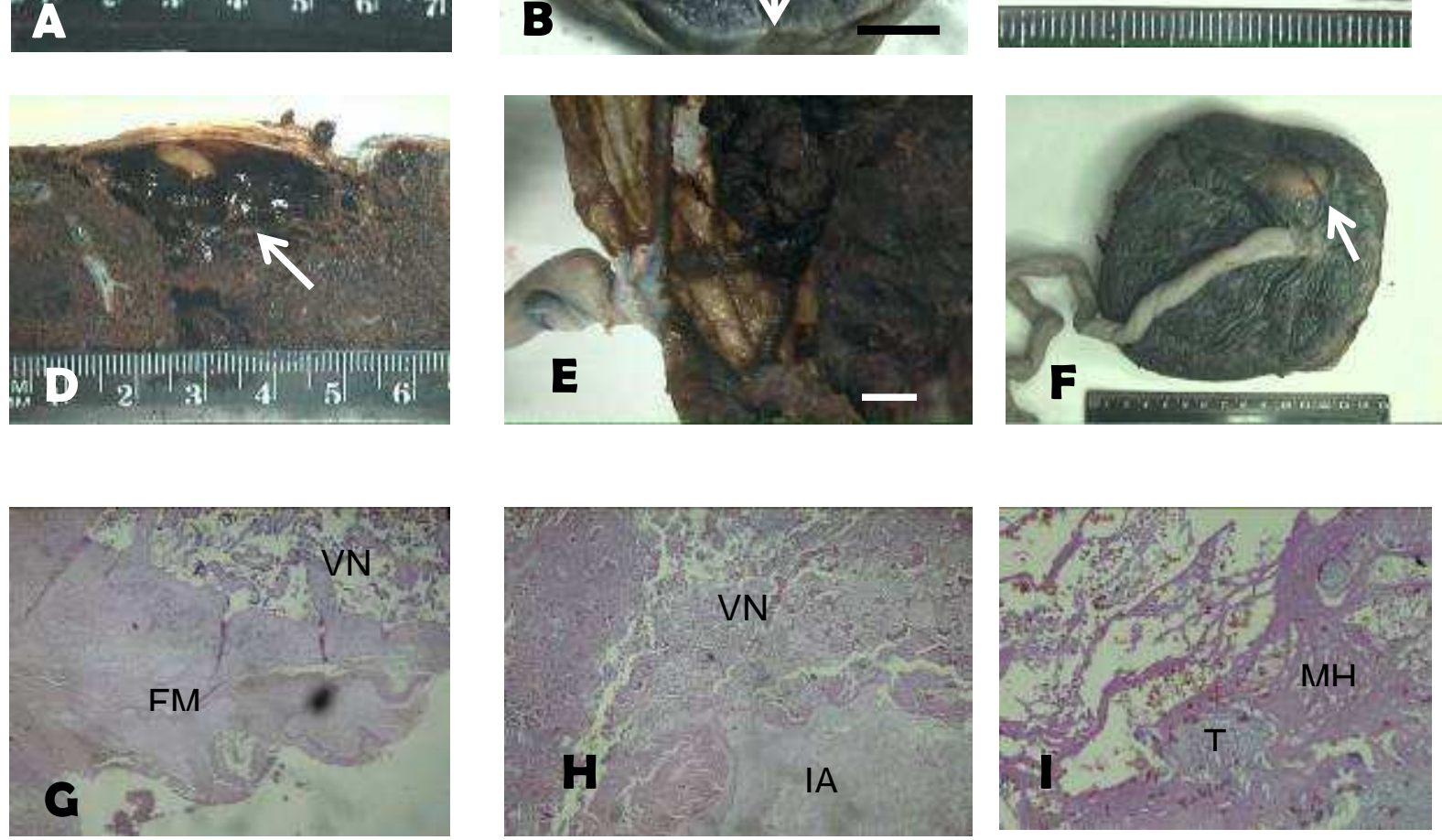

Figura 1: A: Placenta de 29 semanas de gestación con membrana superficial (hialina) depositada en la cara fetal vista en un corte trasversal. B: Fibrosis marginal en una placenta de 30 semanas de gestación, vista desde su cara materna, (escala: $5 \mathrm{~cm}$ ) . C: Corte trasversal en el área marginal de una placenta de 34 semanas de gestación donde se muestran tres infartos antiguos (1, 2, 3). D: Infarto hemorrágico en placenta de 30 semanas de una mujer con eclampsia. E: Inserción velamentosa del cordón umbilical de una placenta de 26 semanas de gestación de una paciente con pre-eclampsia severa (escala: $10 \mathrm{~mm}$ ). F: Zona de infarto antiguo muy cercano a la implantación del cordón umbilical, visto desde la cara fetal, placenta de 31 semanas. G: Se muestra una amplia zona de fibrosis marginal (FM) y vellosidades corales normales (VN), (hematoxilina eosina 100x). $\mathbf{H}$ : vellosidades coriales normales (VN) junto con infarto antiguo (IA), (Hematoxilina eosina $40 x$ ) I: Membrana hialina extensa $(\mathrm{MH})$ junto con nudos de tejido trofoblástico (T) (Hematoxilina eosina 400x).

Revista electrónica publicada por el Departamento de Farmacología de la Escuela de Medicina de la Universidad de Costa Rica, 2060 San José, Costa Rica. @ All rights reserved. 
Tabla 1: Datos del peso placentario según Collen y del producto según Little, respecto a lo observado.

\begin{tabular}{|c|c|c|c|c|c|}
\hline Caso & EG & POP & PEP & PON & PEN \\
\hline 1 & 32 & 323 & 378 & 1020 & 2360 \\
\hline 6 & 29 & 224 & 322 & 825 & 1786 \\
\hline 7 & 31 & 385 & 321 & 1330 & 2073 \\
\hline 8 & 34 & 305 & 389 & 1600 & 2647 \\
\hline 9 & 28 & 489 & 321 & 800 & 1499 \\
\hline 10 & 30 & 390 & 352 & 1250 & 1786 \\
\hline 11 & 29 & 356 & 322 & 1000 & 1786 \\
\hline 13 & 35 & 310 & 432 & 3200 & 2781 \\
\hline 14 & 31 & 209 & 321 & 1850 & 2073 \\
\hline 15 & 35 & 147 & 432 & 2000 & 2781 \\
\hline 18 & 36 & 126 & 436 & 2500 & 2781 \\
\hline 19 & 35 & 249 & 432 & 1900 & 2781 \\
\hline 24 & 36 & 400 & 436 & 2500 & 2781 \\
\hline 25 & 29 & 200 & 322 & 980 & 1786 \\
\hline 26 & 33 & 370 & 350 & 1930 & 2360 \\
\hline 27 & 27 & 170 & 285 & 900 & nd \\
\hline 28 & 34 & 500 & 389 & 2420 & 2647 \\
\hline 30 & 31 & 200 & 321 & 980 & 2073 \\
\hline 32 & 36 & 450 & 436 & 2600 & 2781 \\
\hline 33 & 33 & 250 & 350 & 1520 & 2360 \\
\hline 37 & 35 & 210 & 432 & 2400 & 2781 \\
\hline 38 & 34 & 300 & 389 & 2030 & 2647 \\
\hline 41 & 32 & 290 & 378 & 1500 & 2360 \\
\hline 42 & 33 & 147 & 350 & 1020 & 2073 \\
\hline 43 & 35 & 390 & 432 & 2300 & 2781 \\
\hline 45 & 35 & 250 & 432 & 1600 & 2781 \\
\hline 47 & 33 & 250 & 350 & 2150 & 2073 \\
\hline 48 & 35 & 290 & 430 & 1950 & 2781 \\
\hline
\end{tabular}

EG: Edad gestaciones, POP: peso observado de la placenta, PEP: peso esperado de la placenta, PON: peso observado del niño, PEN: peso esperado del niño.

Revista electrónica publicada por el Departamento de Farmacología de la Escuela de Medicina de la Universidad de Costa Rica, 2060 San José, Costa Rica. ${ }^{\circledR}$ All rights reserved. 
Tabla 2: Distribución del número de placentas, según el diagnóstico y el porcentaje (\%) de área funcional de la placenta.

\begin{tabular}{|c|c|c|c|c|c|c|}
\hline \multirow{2}{*}{$\%$ AFP } & \multirow{2}{*}{$\boldsymbol{N}$} & \multirow{2}{*}{$\mathbf{N}$} & \multicolumn{5}{|c|}{ Diagnóstico } \\
\cline { 3 - 7 } & & & $\begin{array}{c}\text { Pre- } \\
\text { eclampsia } \\
\text { leve }\end{array}$ & $\begin{array}{c}\text { Pre- } \\
\text { eclampsia } \\
\text { severa }\end{array}$ & Eclampsia & HELLP \\
& & & & & & \\
$\mathbf{0 - 2 0}$ & 12 & 43 & 0 & 9 & 2 & $\mathbf{1}$ \\
$\mathbf{2 1 - 4 0}$ & 8 & 29 & 1 & 7 & 0 & $\mathbf{0}$ \\
$\mathbf{4 1 - 6 0}$ & 4 & 14 & 0 & 4 & 0 & $\mathbf{0}$ \\
$\mathbf{6 1 - 8 0}$ & 4 & 14 & 3 & 1 & 0 & $\mathbf{0}$ \\
$\mathbf{8 1 - 1 0 0}$ & 0 & 0 & - & - & - & - \\
\hline total & $\mathbf{2 8}$ & $\mathbf{1 0 0}$ & $\mathbf{4}$ & $\mathbf{2 1}$ & $\mathbf{2}$ & $\mathbf{1}$ \\
\hline
\end{tabular}

\section{Conclusiones}

Se requiere de una segunda etapa de estudio en la cual se realice un estudio clínico más a fondo para poder determinar la naturaleza de los hallazgos en las placentas de nuestro trabajo. Todas las pacientes con insuficiencia placentaria de más de un $50 \%$ presentaron preeclampsia severa. Todas las placentas estudiadas, sin excepción, presentaron cambios que comprometían su eficiencia en su capacidad de barrera madre/feto, por lo que comprometieron importantemente el desarrollo fetal y su estado nutricional Sigue sin conocerse cual es el detonante de la hipertensión durante el embarazo. Se ha correlacionado la presencia de hipertensión inducida por el embrazo con la presencia de vellosidades placentarias defectuosas.

En todas las mujeres que se muestrearon en el presente estudio se encontró que la mayoría de los recién nacidos presentaban bajo peso al nacer.

Revista electrónica publicada por el Departamento de Farmacología de la Escuela de Medicina de la Universidad de Costa Rica, 2060 San José, Costa Rica. ® All rights reserved. 


\section{Bibliografía}

1. Joey P. Granger. Pathophysiology of Hypertension During Preeclampsia Linking Placental Ischemia With endothelial Dysfunction. Hypertension. 2001;38[part 2]:718-722.

2. DeCherney, $\mathrm{H}$, Lauren N. Diagnóstico y tratamiento Ginecoobstétricos. $8^{\mathrm{a}}$ edición. México: Editorial el Manual Moderno, 2003.

3. Catalin S. Buhimschi. Fractional Excretion of Angiogenic Factor in Women With Severe Preeclampsia. Obstetrics \& Gynecology 2006; 107[5]: $1103-1113$

4. Munz C, Holmes $\mathrm{N}$, et al.Human histocompatibility leukocyte antigen[HLA-G] molecules inhibit NKAT3 expressing natural kill cells. J Exp Medicine 1997;185:385-391.

5. Roberts JM, Taylor RM, Goldfiel A. Clinical and biochemical evidence of endothelial cell dysfunction in the pregnancy syndrome preeclampsia. American Journal of Hypertension 1991; 4: 700-708.

6. Mikhail MS, Anyaegbuman A, Garfinkel $D$, et al. Preeclampsia and antioxidant nutrient:decreased plasma levels of reduced ascorbics acid, alpha tocopherol, and geta carotene in women with preeclampsia. American Journal of Obstetrician and Gynecologist 1994:171:150-157.

7. Udainia, A, et al. Morphology Study of Placenta in Pregnancy Induced Hypertension with its Clinical Revelance. J. Anat. Soc. India 2001: 50 [1]; 24-27.

8. Collen L. Dy, et all. Updating reference values for placental weights in Northen Alberta. Am. J. of Obst \& Gyn 2004; 190: 1458-1460.
9. August $P$, and Lindheimer MD. Pathophysiology of preeclampsia. In: Laragh JL, Brenner BM, eds. Hypertension. 2nd ed. New York, NY: Raven Press; 1995:2407-2426.

10. Brosens IA, Robertson WB, Dixon HG. The role of the spiral arteries in the pathogenesis of preeclampsia. Obstet Gynecol Ann. 1972;1: 177-191.

11. Hanretty KP, Whittle MJ, Rubin PC. Doppler uteroplacental waveforms in pregnancy-induced hypertension: a reappraisal. Lancet. 1988;1: 850-852.

12. Fisher SJ, Roberts JM. Defects in placentation and placental perfusion. In: Linhheimer MD, Roberts JM, Cunningham FG, eds. Chesley's Hypertensive Disorders in Pregnancy. 2nd ed. Stanford, CT: Appleton \& Lange; 1999:377-394.

13. Taylor RN, Roberts JM. Endothelial cell dysfunction. In: Linhheimer MD, Roberts JM, Cunningham FG, eds. Chesley's Hypertensive Disorders in Pregnancy. 2nd ed. Stanford, CT: Appleton \& Lange; 1999: 395-429.

14. Roberts JM., Taylor RN, Goldfien A. Clinical and biochemical evidence of endothelial cell dysfunction in the pregnancy syndrome preeclampsia. Am J Hypertens. 1991;4:700 -708.

15. Carstens R. Mario, et all. Síndrome hipertensivo del embarazo. Universidad Pontificia Católica de Chile. Boletín perinatal nov 2001.

16. Little. Ruth. Placental weight and its ratio to birthweight in an Ukranainian city. Earlu Human Development 71 [2003] 117-127.

Revista electrónica publicada por el Departamento de Farmacología de la Escuela de Medicina de la Universidad de Costa Rica, 2060 San José, Costa Rica. $\circledR$ All rights reserved. 


\section{Correspondencia:}

\section{Luis E. Zamora Vargas}

Correo electrónico: luiszamora08@gmail.com

Departamento Anatomía Escuela Medicina UCR.

Teléfono (506) 2207-4488.

Revista electrónica publicada por el Departamento de Farmacología de la Escuela de Medicina de la Universidad de Costa Rica, 2060 San José, Costa Rica. ${ }^{\circledR}$ All rights reserved. 